\title{
MACRONUTRIENTS IN THE Eucalyptus Dunnii Maiden ROWS SUBMITTED TO POTASSIUM FERTILIZATION IN VARIABLE CHARGE SOILS ${ }^{1}$
}

\author{
Thays Schneider ${ }^{2 *}$, Adriel Ferreira da Fonseca ${ }^{3}$ and Laíse da Silveira Pontes ${ }^{4}$
}

\footnotetext{
${ }^{1}$ Received on 15.09.2016 accepted for publication on 31.10.2017.

${ }^{2}$ Universidade Estadual de Ponta Grossa, Doutora em Agronomia, Ponta Grossa, PR-Brasil. E-mail:<thaysschneider12@gmail.com>.

${ }^{3}$ Universidade Estadual de Ponta Grossa, Departamento de Ciência do Solo e Engenharia Agrícola, Ponta Grossa, PR-Brasil. E-mail:<adriel@uepg.br>.

${ }^{4}$ Instituto Agronômico do Paraná, Ponta Grossa, PR- Brasil. E-mail: <laisepontes@iapar.br>.

*Corresponding author.
}

\begin{abstract}
The contribution of nutrient cycling from eucalyptus residues in an Integrated Crop-Livestock System (ICLS) extracting potassium (K) in deeper layers and releasing on the surface should be further investigated in soils with variable charge. The objectives of this study were to evaluate the effects of $\mathrm{K}$ rates $(0,100$, 200 and $300 \mathrm{~kg} \mathrm{ha}^{-1} \mathrm{yr}^{-1}$ of $\mathrm{K}_{2} \mathrm{O}$ ) applied on the soil surface in the entire area in rows of ICLS with eucalyptus, and relate these doses to macronutrient levels in the soil. The implemented experimental design was a randomized complete block using a split plot with three replications. The plot consisted of two rows and subplot by $\mathrm{K}$ rates. Soil fertility attributes were conducted at 6,12 and 30 months after the start of the experiment. Medium to high concentrations of $\mathrm{K}$ were observed in profile up to 12 months, and very low concentrations of $\mathrm{K}$ were observed in the profile 30 months after the experiment was initiated. High concentrations of $\mathrm{Ca}, \mathrm{Mg}$ were observed throughout the profile favoring saturation in an effective cation exchange capacity (ECEC). However, high K saturation in ECEC was observed at 6 and 12 months, and this decreased at 30 months of study. No leaching was observed, although acidity was high.
\end{abstract}

Keywords: Agrosilvopastoral system, nutrient cycling, Typic Hapludox soil.

\section{MACRONUTRIENTES EM RENQUES DE EuCALYPTUS Dunnii Maiden SUBMETIDOS À ADUBAÇ̃̃o POTÁSSICA EM SOLOS DE CARGA VARIÁVEL}

\begin{abstract}
RESUMO - A contribuição da ciclagem de nutrientes provenientes dos resíduos de eucalipto em sistema integrado de produção agropecuária (SIPA), extraindo o potássio $(K)$ de camadas mais profundas e liberandoo na superficie, merece ser melhor investigada em solos com carga variável. Os objetivos deste trabalho foram avaliar os efeitos de doses de $K\left(0,100,200\right.$ e $300 \mathrm{~kg} \mathrm{ha}^{-1}$ ano $^{-1}$ de $\left.K_{2} \mathrm{O}\right)$ aplicadas na superficie do solo, em área total, nos renques de um SIPA com eucalipto, e relacionar estas doses com os teores de macronutrientes no solo. O delineamento experimental adotado foi o de blocos completos casualizados com parcela subdividida e três repetições. A parcela foi composta por dois renques e a subparcela pelas doses de K. Os atributos de fertilidade do solo foram realizados aos 6, 12 e 30 meses após o início do experimento. Concentrações de média a alta de K no perfil foram verificadas até 12 meses e concentrações baixas foram observadas após 30 meses do início do experimento. Altas concentrações de Ca e Mg foram verificadas no perfil estudado, favorecendo a saturação destes na capacidade de troca de cátions efetiva (CTCe). A saturação do K na CTCe reduziu aos 30 meses, apesar da adequada saturação aos 6 e 12 meses. Não foi observada lixiviação, embora a acidez fosse alta.
\end{abstract}

Palavras-Chave: Integração lavoura-pastagem-floresta; ciclagem de nutrientes; Latossolo Vermelho distrófico. 


\section{INTRODUCTION}

The soils originated by Furnas Formation belonging to the Second Paraná Plateau are composed of shales, predominantly quartzite and medium to coarse granulation quartz (Retzlaf et al., 2006), which originate soils of low fertility (Reissmann and Wisniewski, 2000). In this context, different nutrient contents in the source material may influence the composition of tree species within the landscape unit (Foster and Bhatti, 2006).

The tree component of an Integrated Crop-Livestock System (ICLS) provides a differentiated microclimate, resulting in a decrease in the radiation percentage, a decrease in air and soil temperature, an increase in the percentage of humidity in the air, a decrease in evapotranspiration, and improvement in maintenance of soil moisture (Menezes et al., 2002; Bernardino and Garcia, 2009). This last factor has been determinant in the availability of $\mathrm{K}$ to the plants because the movement of the soil solution can be driven by differences in water potential (mass flow) or by differences in concentration (diffusion). When the concentration of $\mathrm{K}$ in the soil solution is high, mass flux is the predominant ion-root contact mechanism; on the other hand, diffusion is the dominant ion-root contact mechanism in the transport of $\mathrm{K}$ to the roots at low concentrations (Oliveira et al., 2004; Malavolta, 2005; Marschner, 2012).

The contribution of eucalyptus residues to $\mathrm{K}$ release is large (Cunha et al., 2005) due to its ability to absorb it from deeper layers (below $20 \mathrm{~cm}$ ) and then release it into the superficial layer $(0-20 \mathrm{~cm})$ (Witschoreck et al., 2003; Freycon et al., 2015). The amount of K available in the first $20 \mathrm{~cm}$ of most soils has been insufficient to supply the average demand for eucalyptus (Silveira et al., 2005). On the other hand, annual crop fertilization in an ICLS gradually changes the chemistry of exchange complex, mainly in the $0-20 \mathrm{~cm}$ layer, with elevation of the cation exchange capacity (CEC), organic carbon (OC), and effective cation exchange capacity (ECEC). With this, the residual effect of fertilization increases, which may reduce the need of several applications during the eucalyptus production cycle (Silveira et al., 2005).

Considering the above, it is expected that potassium fertilization will increase losses by $\mathrm{K}$ leaching due to high mobility and $\mathrm{K}$ biochemical cycling. The objective of this study was to understand the effects of potassium fertilization $\left(0,100,200\right.$ and $\left.300 \mathrm{~kg} \mathrm{ha}^{-1} \mathrm{yr}^{-1} \mathrm{~K}_{2} \mathrm{O}\right)$ on

Revista Árvore. 2018;42(1):e420117 the dynamics of macronutrients in variable charge soil in rows of an ICLS with Eucalyptus dunnii.

\section{MATERIALAND METHODS}

The study was conducted at the Experimental Station Farm Model of the Paraná Agronomic Institute (Latitude: $25^{\circ} 06^{\prime} 24^{\prime \prime} \mathrm{S}$, longitude: $50^{\circ} 02^{\prime} 35^{\text {' }} \mathrm{W}$ and average elevation: 1015 meters), Ponta Grossa (PR), Brazil. The cumulative rainfall of 2012-2014 was 1700, 1706, $1614 \mathrm{~mm}$ respectively, and of $748 \mathrm{~mm}$ for the summer and fall of 2015 . The average annual temperature of 2012-2014 was 18.5; 17.8; and $18.7^{\circ} \mathrm{C}$ respectively, and $19.1^{\circ} \mathrm{C}$ for the summer and fall of 2015. The data were obtained from a SIMEPAR Meteorological Station (25135001) in the Ponta Grossa region. The climate of the region according to the Köppen classification is Cfb type, humid subtropical mesothermic. The main chemical and physical attributes of the soil in the rows collected in May 2012 before the application of $\mathrm{K}$ doses in the 0 to $60 \mathrm{~cm}$ layer were: $\mathrm{pH}\left(\mathrm{CaCl}_{2}\right) 4.4$, OC $16.9 \mathrm{~g} \mathrm{dm}^{-3}, 16.0 ; 15.4 ; 1.4 \mathrm{mmol}_{\mathrm{c}} \mathrm{dm}^{-3}$ of Ca, $\mathrm{Mg}$, $\mathrm{K}$, respectively; as well as $580.2 ; 116.5$ and $303.3 \mathrm{~g} \mathrm{~kg}^{-1}$ of sand, silt and clay, respectively.

The experimental design was a randomized block with subdivided plots and three replications. The 24 plots of $24 \mathrm{~m}^{2}$ each consisted of rows 1 and 2 and the subplots of doses $0,100,200$ and $300 \mathrm{~kg} \mathrm{ha}^{-1}$ year-1 of $^{-1}$ $\mathrm{K}_{2} \mathrm{O}$ in $\mathrm{KCl}$ form applied to the soil surface. The rows were subdivided due to a distance between each other of $20 \mathrm{~m}$ and the greater slope of row 1 in relation to row 2 (smoothly undulating relief). The eucalyptus seedlings were planted in 2007 in a double-lane, eastwest direction, transversely the terrain slope and spaced 3 meters between plants and 4 meters between rows. The K doses were split into two applications; half in the fall (April), and the other half in the spring (October), meaning at the moment of winter crops (white oats) and summer crops (soybean or corn) respectively, which was another experiment analyzed concomitantly to the present study.

Twelve (12) single samples of the 0-5, 5-10, 10$15,15-20 \mathrm{~cm}$ layers and three simple samples of the 20-40 and 40-60 cm layers were collected using a gouge and dutch auger, respectively, for determining $\mathrm{OC}, \mathrm{pH}$, $\mathrm{H}+\mathrm{Al}, \mathrm{K}, \mathrm{Ca}$, and $\mathrm{Mg}$ to form a composed sample by subplot. The dry soil samples were analyzed to determine the following attributes: $\mathrm{pH}, \mathrm{H}+\mathrm{Al}, \mathrm{K}, \mathrm{Ca}$, $\mathrm{Mg}$ and Al (Pavan et al., 1992), as well as OC by the Walkley-Black method (Cantarella et al., 2001). The

\section{Revista Árvore. 2018;42(1):e420117}


sum of bases (SB), potential and effective cation exchange capacity (CEC and ECEC), base saturation(V\%), aluminum saturation ( $\mathrm{m} \%$ ) and $\mathrm{K}, \mathrm{Ca}$ and $\mathrm{Mg}$ saturation in ECEC were calculated (KECEC, CaECEC and MgECEC respectively) according to Pavan et al. (1992).

The results were submitted to the Bartlet analysis, the data were transformed to obtain homogeneous variances when significant, and then variance analysis (ANOVA) was calculated. Regression equations were elaborated when ANOVA was significant, adopting the coefficient of determination as the criterion of choice, and Tukey's test $(\mathrm{p}<0.05)$ for the means comparison of the soil concentrations between rows using Statgraphics ${ }^{\circledR}$ Centurion Software.

\section{RESULTS}

\subsection{Soil attributes at 6 months}

There were no significant interactions between row and dose for soil attributes. Differences between rows $(\mathrm{p}<0.05)$ were observed for SB in the $0-5 \mathrm{~cm}$ layer,
$\mathrm{Mg}$ and $\mathrm{SB}$ in the $5-10 \mathrm{~cm}$ layer, $\mathrm{Mg}$ in the $10-15 \mathrm{~cm}$ and $15-20 \mathrm{~cm}$ layers, and KECEC in the 40-60 cm layer (Table 1). Significant dose effects for $\mathrm{Mg}$ and KECEC in the $0-5 \mathrm{~cm}$ layer, $\mathrm{K}$ in the $5-10 \mathrm{~cm}$ layer (Figure 1) and CEC in the 20-40 cm layer (Table 4) were observed.

\subsection{Soil attributes at 12 months}

Interactions were observed for the ECEC and SB attributes in the layer of $0-5-\mathrm{cm}$, and CaECEC in the 20-40 cm layer (Table 4). A significant effect of the rows was only observed for SB in the $15-20 \mathrm{~cm}$ layer, and $\mathrm{CaECEC}, \mathrm{V} \%$ and $\mathrm{m} \%$ in the $20-40 \mathrm{~cm}$ layer (Table 2). The dose effect was significant for K, ECEC, SB and KECEC in the 0-5 cm layer (Table 4), $\mathrm{K}$ and KECEC in the $5-10 \mathrm{~cm}$ layer (Figure 1), K and KECEC in the 10-15 cm layer (Table 4).

\subsection{Soil attributes at 30 months}

No interactions were observed for the analyzed variables. Differences between rows were observed for $\mathrm{pH}$ in the $10-15 \mathrm{~cm}$ layer, along with $\mathrm{pH}, \mathrm{CEC}$ and

Table 1 - Average concentrations of soil chemical attributes in rows submitted to the K dose treatments 6 months after the start of the experiment.

Tabela 1 - Concentrações médias dos atributos químicos do solo nos renques submetidos aos tratamentos de doses de $K$, aos 6 meses após o início do experimento.

\begin{tabular}{|c|c|c|c|c|c|c|c|c|c|c|c|c|c|c|}
\hline Layer & ion & $\overline{\mathrm{OC}}$ & $\mathrm{pH} \mathrm{CaCl}{ }_{2}$ & $\mathrm{Ca}$ & $\mathrm{Mg}$ & $\mathrm{K}$ & CEC & ECEC & SB & KECEC & CaECEC & MgECEC & $\mathrm{V}$ & $\mathrm{m}$ \\
\hline $\mathrm{cm}$ & & & $\mathrm{g} \mathrm{dm}^{-3}$ & & & & $\mathrm{amol}_{\mathrm{c}} \mathrm{dm}$ & & & & & $\%$ & & \\
\hline \multirow[t]{3}{*}{$0-5$} & Row 1 & $24.0 \mathrm{a}$ & $5.0 \mathrm{a}$ & $34.5 \mathrm{a}$ & $23.1 \mathrm{a}$ & $2.3 \mathrm{a}$ & $130.9 \mathrm{a}$ & $60.5 \mathrm{a}$ & $59.9 \mathrm{~b}$ & $3.8 \mathrm{a}$ & $57.3 \mathrm{a}$ & $37.9 \mathrm{a}$ & $46.3 \mathrm{a}$ & $1.0 \mathrm{a}$ \\
\hline & & $25.5 \mathrm{a}$ & $5.1 \mathrm{a}$ & $35.1 \mathrm{a}$ & $24.3 \mathrm{a}$ & $2.3 \mathrm{a}$ & $130.0 \mathrm{a}$ & $62.2 \mathrm{a}$ & $61.8 \mathrm{a}$ & $3.8 \mathrm{a}$ & $57.0 \mathrm{a}$ & $38.6 \mathrm{a}$ & & $0.6 \mathrm{a}$ \\
\hline & & 9.9 & 6.4 & 14.0 & 28.2 & 24.1 & 12.6 & 12.6 & 12.5 & 27.2 & 13.9 & 21.6 & 13.9 & 166.6 \\
\hline \multirow[t]{3}{*}{$5-10$} & Row & $20.4 \mathrm{a}$ & $4.5 \mathrm{a}$ & $13.7 \mathrm{a}$ & $17.71 \mathrm{~b}$ & $2.0 \mathrm{a}$ & $121.9 \mathrm{a}$ & $38.3 \mathrm{a}$ & $34.0 \mathrm{~b}$ & $5.8 \mathrm{a}$ & $34.4 \mathrm{a}$ & $48.0 \mathrm{a}$ & $27.3 \mathrm{a}$ & $11.8 \mathrm{a}$ \\
\hline & & $.0 \mathrm{a}$ & & $15.5 \mathrm{a}$ & $21.07 \mathrm{a}$ & $2.0 \mathrm{a}$ & $126.9 \mathrm{a}$ & $41.8 \mathrm{a}$ & $38.5 \mathrm{a}$ & $4.9 \mathrm{a}$ & $36.4 \mathrm{a}$ & $50.5 \mathrm{a}$ & & \\
\hline & $\mathrm{CV}^{\circ}$ & 12.0 & 5.3 & 42.0 & 32.8 & 25.6 & 19.8 & 27.9 & 28.2 & 45.1 & 25.5 & 21.2 & 21.8 & 62.9 \\
\hline \multirow[t]{3}{*}{$10-15$} & ow 1 & $14.4 \mathrm{a}$ & $4.2 \mathrm{a}$ & $8.8 \mathrm{a}$ & $30.7 \mathrm{a}$ & $1.3 \mathrm{a}$ & $141.9 \mathrm{a}$ & $50.8 \mathrm{a}$ & $40.8 \mathrm{a}$ & $3.7 \mathrm{a}$ & $22.4 \mathrm{a}$ & $50.2 \mathrm{a}$ & $25.0 \mathrm{a}$ & $23.8 \mathrm{a}$ \\
\hline & ow 2 & $16.2 \mathrm{a}$ & $4.3 \mathrm{a}$ & $9.5 \mathrm{a}$ & $18.6 \mathrm{~b}$ & $1.3 \mathrm{a}$ & $128.4 \mathrm{a}$ & $37.5 \mathrm{a}$ & $29.4 \mathrm{a}$ & $3.6 \mathrm{a}$ & $24.6 \mathrm{a}$ & $49.5 \mathrm{a}$ & $22.8 \mathrm{a}$ & 22.3 \\
\hline & $\mathrm{CV} \%$ & 18.1 & 6.2 & 59.9 & 134.1 & 26.8 & 27.7 & 74.1 & 91.5 & 51.7 & 48.2 & 23.5 & 41.2 & 45.8 \\
\hline \multirow[t]{3}{*}{$15-20$} & ow 1 & $14.3 \mathrm{a}$ & $4.1 \mathrm{a}$ & $4.9 \mathrm{a}$ & $9.4 \mathrm{~b}$ & $1.3 \mathrm{a}$ & $118.0 \mathrm{a}$ & $27.3 \mathrm{a}$ & $15.6 \mathrm{a}$ & $5.2 \mathrm{a}$ & $17.4 \mathrm{a}$ & $36.7 \mathrm{a}$ & $13.6 \mathrm{a}$ & 40.9 \\
\hline & & $15.4 \mathrm{a}$ & $4.0 \mathrm{a}$ & $5.1 \mathrm{a}$ & $11.8 \mathrm{a}$ & $1.1 \mathrm{a}$ & $123.2 \mathrm{a}$ & $29.7 \mathrm{a}$ & $18.0 \mathrm{a}$ & $4.0 \mathrm{a}$ & $16.8 \mathrm{a}$ & $39.0 \mathrm{a}$ & $14.6 \mathrm{a}$ & 40.3 \\
\hline & & 16.0 & 4.7 & 72.5 & 37.0 & 23.3 & 17.6 & 23.8 & 33.1 & 41.8 & 59.1 & 27.5 & 30.0 & 35.0 \\
\hline \multirow[t]{3}{*}{$20-40$} & Row 1 & $16.6 \mathrm{a}$ & $4.1 \mathrm{a}$ & $7.5 \mathrm{a}$ & $16.4 \mathrm{a}$ & $1.0 \mathrm{a}$ & $132.4 \mathrm{a}$ & $39.0 \mathrm{a}$ & $25.0 \mathrm{a}$ & $2.6 \mathrm{a}$ & $19.4 \mathrm{a}$ & $41.3 \mathrm{a}$ & $18.8 \mathrm{a}$ & $36.7 \mathrm{a}$ \\
\hline & Row 2 & $18.0 \mathrm{a}$ & $4.1 \mathrm{a}$ & $9.9 \mathrm{a}$ & $16.4 \mathrm{a}$ & $1.1 \mathrm{a}$ & $137.0 \mathrm{a}$ & $41.2 \mathrm{a}$ & $27.4 \mathrm{a}$ & $2.8 \mathrm{a}$ & $22.6 \mathrm{a}$ & $40.2 \mathrm{a}$ & $20.0 \mathrm{a}$ & 34.4 \\
\hline & & 13.3 & 2.3 & 85.1 & 41.1 & 42.6 & 9.5 & 21.1 & 29.4 & 44.6 & 81.3 & 36.9 & 28.2 & 24.4 \\
\hline \multirow[t]{3}{*}{$40-60$} & & $.9 \mathrm{a}$ & & 1.0 & & $0.7 \mathrm{a}$ & $112.0 \mathrm{a}$ & $25.0 \mathrm{a}$ & $10.3 \mathrm{a}$ & $2.9 \mathrm{~b}$ & $3.9 \mathrm{a}$ & $33.7 \mathrm{a}$ & & 59.6 \\
\hline & & $14.5 \mathrm{a}$ & $4.2 \mathrm{a}$ & $1.1 \mathrm{a}$ & $9.7 \mathrm{a}$ & $0.6 \mathrm{a}$ & $115.1 \mathrm{a}$ & $25.8 \mathrm{a}$ & $11.4 \mathrm{a}$ & $2.4 \mathrm{a}$ & $4.1 \mathrm{a}$ & $37.6 \mathrm{a}$ & $10.0 \mathrm{a}$ & 55.9 \\
\hline & & 26.9 & 1.3 & 76.3 & 32.6 & 42.5 & 8.7 & 11.6 & 29.2 & 46.0 & 67.3 & 27.8 & 27.2 & 16.6 \\
\hline
\end{tabular}

Transformations made: COSR(C_B); COSR(C_F); 1/pH_C; DIFF(pH_D); COSR(Ca_D); COSR(Ca_E);COSR(Mg_C); COSR(Mg_D); COSR(CEC_C); COSR(ECEC_C); 1/SB_C; COSR(SB_E $)$; COSR(SB_C); COSR(KECEC_E); COSR (KECEC_F); COSR(CaECEC_E); $\operatorname{COSR}(\mathrm{MgE} \overline{\mathrm{CEC}} \mathrm{C})$; $\operatorname{COSR}(\mathrm{MgECEC} \overline{\mathrm{E}}) ; \operatorname{COSR}(\mathrm{V} \% \overline{\mathrm{C}})$. The presented means for each transformed variable refer to the original values and the comparison of means test to the transformed variables. Means followed by the same letter for each layer do not differ from each other by the Tukey test when $\mathrm{p}<0.05$. 

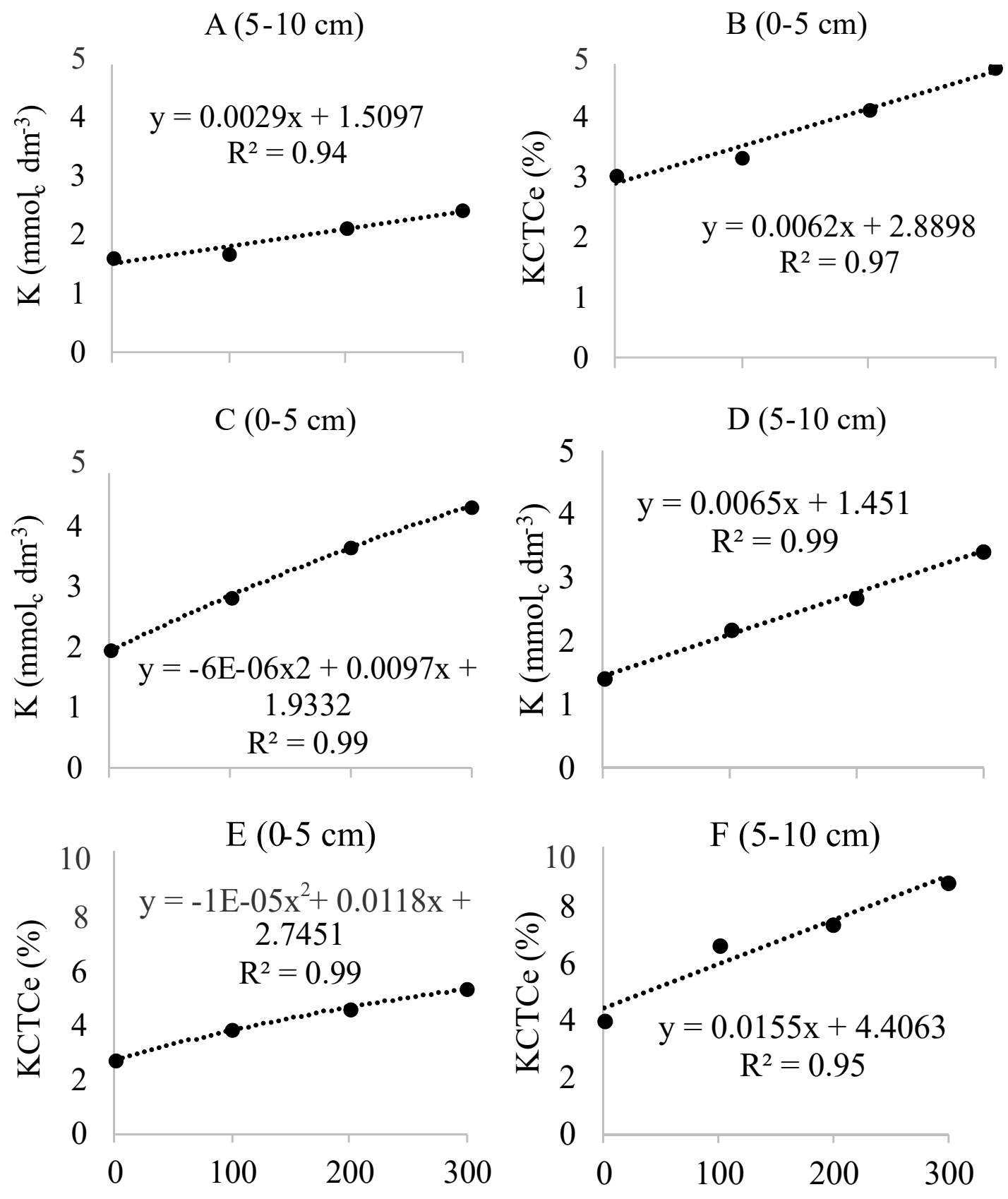

$K_{2} O$ Doses $\left(\mathrm{kg} \mathrm{ha}^{-1}\right.$ year $\left.^{-1}\right)$

Figure 1-Potassium concentration (K) in the 5-10 cm layer (1A), percentage of potassium in the effective cation exchange capacity (KECEC) in the 0-5 cm layer (1B) at 6 months, $K$ in the $0-5 \mathrm{~cm}(1 \mathrm{C})$ and $5-10 \mathrm{~cm}$ layers (1D), KECEC in the $0-5 \mathrm{~cm}$ layer $(1 \mathrm{E})$ and $\mathrm{KECEC}$ in the $5-10 \mathrm{~cm}$ layer $(1 \mathrm{~F})$ at 12 months after application of $\mathrm{K}_{2} \mathrm{O}$ rates. Figura 1 - Concentração de potássio $(K)$ na camada de $5-10 \mathrm{~cm}(1 \mathrm{~A})$, percentual de potássio na capacidade de troca cationica efetiva (KCTCe) na camada de $0-5 \mathrm{~cm}$ (1B) aos 6 meses, concentração de potássio na camada de $0-5 \mathrm{~cm}$ (1C) e 5-10 cm (1D), KCTCe na camada de 0-5 cm (1E) e KCTCe na camada de 5-10 cm (1F) do solo, aos 12 meses após aplicação das doses de $\mathrm{K}_{2} \mathrm{O}$.

Revista Árvore. 2018;42(1):e420117 
Table 2 - Average concentrations of soil chemical attributes in rows submitted to the K dose treatments 12 months after the start of the experiment.

Tabela 2 - Concentrações médias dos atributos químicos do solo nos renques submetidos aos tratamentos de doses de $K$, aos 12 meses após o início do experimento.

\begin{tabular}{|c|c|c|c|c|c|c|c|c|c|c|c|c|c|c|}
\hline Layer & Variation & $\mathrm{OC}$ & $\underset{\mathrm{CaCl}_{2}}{\mathrm{pH}}$ & $\mathrm{Ca}$ & $\mathrm{Mg}$ & K & CEC & ECEC & SB & KECEC & CaECEC & MgECEC & $\mathrm{V}$ & $\mathrm{m}$ \\
\hline $\mathrm{cm}$ & & $\mathrm{g} \mathrm{dm}^{-3}$ & & & \multicolumn{5}{|c|}{$\mathrm{mmol}_{\mathrm{c}} \mathrm{dm}^{-3}$} & & & $\%$ & & \\
\hline $0-5$ & $\begin{array}{c}\text { Row } 1 \\
\text { Row } 2 \\
\text { CV\% }\end{array}$ & $\begin{array}{c}24.6 \mathrm{a} \\
25.3 \mathrm{a} \\
9.9\end{array}$ & $\begin{array}{c}4.8 \mathrm{a} \\
4.8 \mathrm{a} \\
4.6\end{array}$ & $\begin{array}{c}35.5 \mathrm{a} \\
38.1 \mathrm{a} \\
14.7\end{array}$ & $\begin{array}{c}35.3 \mathrm{a} \\
36.5 \mathrm{a} \\
16.6 \\
\end{array}$ & $\begin{array}{l}3.2 \mathrm{a} \\
3.2 \mathrm{a} \\
32.3\end{array}$ & $\begin{array}{c}129.8 \mathrm{a} \\
136.4 \mathrm{a} \\
8.0\end{array}$ & $\begin{array}{c}74.7 \mathrm{a} \\
78.4 \mathrm{a} \\
10.9 \\
\end{array}$ & $\begin{array}{c}73.9 \mathrm{a} \\
77.8 \mathrm{a} \\
11.2\end{array}$ & $\begin{array}{l}4.2 \mathrm{a} \\
4.1 \mathrm{a} \\
27.7 \\
\end{array}$ & $\begin{array}{c}47.6 \mathrm{a} \\
48.8 \mathrm{a} \\
11.3 \\
\end{array}$ & $\begin{array}{c}47.3 \mathrm{a} \\
46.3 \mathrm{a} \\
11.0\end{array}$ & $\begin{array}{c}57.1 \mathrm{a} \\
57.2 \mathrm{a} \\
10.6 \\
\end{array}$ & $\begin{array}{l}1.0 \mathrm{a} \\
0.8 \mathrm{a} \\
91.1 \\
\end{array}$ \\
\hline $5-10$ & $\begin{array}{c}\text { Row } 1 \\
\text { Row } 2 \\
\text { CV\% } \\
\end{array}$ & $\begin{array}{c}20.8 \mathrm{a} \\
20.4 \mathrm{a} \\
14.3 \\
\end{array}$ & $\begin{array}{c}4.4 \mathrm{a} \\
4.4 \mathrm{a} \\
5.3\end{array}$ & $\begin{array}{c}14.7 \mathrm{a} \\
14.6 \mathrm{a} \\
25.1 \\
\end{array}$ & $\begin{array}{c}15.5 \mathrm{a} \\
16.8 \mathrm{a} \\
26.4\end{array}$ & $\begin{array}{c}2.5 \mathrm{a} \\
2.4 \mathrm{a} \\
36.6 \\
\end{array}$ & $\begin{array}{c}104.3 \mathrm{a} \\
106.6 \mathrm{a} \\
9.5\end{array}$ & $\begin{array}{c}35.9 \mathrm{a} \\
36.3 \mathrm{a} \\
13.8 \\
\end{array}$ & $\begin{array}{c}32.7 \mathrm{a} \\
33.8 \mathrm{a} \\
20.1 \\
\end{array}$ & $\begin{array}{l}7.0 \mathrm{a} \\
6.6 \mathrm{a} \\
33.2\end{array}$ & $\begin{array}{c}40.7 \mathrm{a} \\
39.8 \mathrm{a} \\
19.0 \\
\end{array}$ & $\begin{array}{c}42.3 \mathrm{a} \\
46.3 \mathrm{a} \\
22.6\end{array}$ & $\begin{array}{c}31.8 \mathrm{a} \\
32.3 \mathrm{a} \\
25.8 \\
\end{array}$ & $\begin{array}{c}10.1 \mathrm{a} \\
7.3 \mathrm{a} \\
88.7 \\
\end{array}$ \\
\hline $10-15$ & $\begin{array}{c}\text { Row } 1 \\
\text { Row } 2 \\
\text { CV\% } \\
\end{array}$ & $\begin{array}{c}17.2 \mathrm{a} \\
19.2 \mathrm{a} \\
17.4 \\
\end{array}$ & $\begin{array}{c}4.0 \mathrm{a} \\
4.1 \mathrm{a} \\
5.6 \\
\end{array}$ & $\begin{array}{c}8.2 \mathrm{a} \\
8.1 \mathrm{a} \\
58.0 \\
\end{array}$ & $\begin{array}{c}12.7 \mathrm{a} \\
11.9 \mathrm{a} \\
32.4 \\
\end{array}$ & $\begin{array}{l}1.9 \mathrm{a} \\
1.8 \mathrm{a} \\
34.9 \\
\end{array}$ & $\begin{array}{c}108.8 \mathrm{a} \\
114.3 \mathrm{a} \\
9.7\end{array}$ & $\begin{array}{c}31.1 \mathrm{a} \\
30.1 \mathrm{a} \\
14.9 \\
\end{array}$ & $\begin{array}{c}22.9 \mathrm{a} \\
21.7 \mathrm{a} \\
32.3 \\
\end{array}$ & $\begin{array}{l}6.3 \mathrm{a} \\
5.8 \mathrm{a} \\
30.2 \\
\end{array}$ & $\begin{array}{c}25.8 \mathrm{a} \\
26.3 \mathrm{a} \\
48.8 \\
\end{array}$ & $\begin{array}{c}40.1 \mathrm{a} \\
39.5 \mathrm{a} \\
21.5 \\
\end{array}$ & $\begin{array}{c}21.2 \mathrm{a} \\
19.5 \mathrm{a} \\
36.4 \\
\end{array}$ & $\begin{array}{c}27.9 \mathrm{a} \\
28.4 \mathrm{a} \\
52.7 \\
\end{array}$ \\
\hline $15-20$ & $\begin{array}{c}\text { Row } 1 \\
\text { Row } 2 \\
\text { CV\% }\end{array}$ & $\begin{array}{c}16.3 \mathrm{a} \\
17.6 \mathrm{a} \\
10.6 \\
\end{array}$ & $\begin{array}{c}4.0 \mathrm{a} \\
4.0 \mathrm{a} \\
3.1 \\
\end{array}$ & $\begin{array}{c}3.9 \mathrm{a} \\
3.2 \mathrm{a} \\
75.0\end{array}$ & $\begin{array}{l}6.7 \mathrm{a} \\
6.9 \mathrm{a} \\
26.1 \\
\end{array}$ & $\begin{array}{l}1.3 \mathrm{a} \\
1.2 \mathrm{a} \\
36.9\end{array}$ & $\begin{array}{c}112.5 \mathrm{a} \\
117.3 \mathrm{a} \\
12.6\end{array}$ & $\begin{array}{c}22.7 \mathrm{a} \\
23.5 \mathrm{a} \\
15.3 \\
\end{array}$ & $\begin{array}{c}11.8 \mathrm{~b} \\
11.2 \mathrm{a} \\
35.3 \\
\end{array}$ & $\begin{array}{l}5.6 \mathrm{a} \\
5.1 \mathrm{a} \\
35.8\end{array}$ & $\begin{array}{c}16.4 \mathrm{a} \\
13.5 \mathrm{a} \\
67.3 \\
\end{array}$ & $\begin{array}{c}29.3 \mathrm{a} \\
29.4 \mathrm{a} \\
18.2\end{array}$ & $\begin{array}{c}10.9 \mathrm{a} \\
9.9 \mathrm{a} \\
43.6\end{array}$ & $\begin{array}{c}48.8 \mathrm{a} \\
52.0 \mathrm{a} \\
27.3 \\
\end{array}$ \\
\hline $20-40$ & $\begin{array}{c}\text { Row } 1 \\
\text { Row } 2 \\
\text { CV\% }\end{array}$ & $\begin{array}{c}14.9 \mathrm{a} \\
15.8 \mathrm{a} \\
18.8\end{array}$ & $\begin{array}{c}3.9 \mathrm{a} \\
3.9 \mathrm{a} \\
4.3 \\
\end{array}$ & $\begin{array}{l}1.8 \mathrm{a} \\
2.3 \mathrm{a} \\
169.0\end{array}$ & $\begin{array}{l}5.5 \mathrm{a} \\
4.5 \mathrm{a} \\
48.8\end{array}$ & $\begin{array}{l}1.1 \mathrm{a} \\
1.2 \mathrm{a} \\
47.4\end{array}$ & $\begin{array}{c}115.9 \mathrm{a} \\
126.4 \mathrm{a} \\
10.3\end{array}$ & $\begin{array}{c}20.6 \mathrm{a} \\
20.7 \mathrm{a} \\
16.6 \\
\end{array}$ & $\begin{array}{c}8.5 \mathrm{a} \\
8.0 \mathrm{a} \\
72.6\end{array}$ & $\begin{array}{l}5.1 \mathrm{a} \\
5.6 \mathrm{a} \\
37.1 \\
\end{array}$ & $\begin{array}{l}8.2 \mathrm{~b} \\
9.2 \mathrm{a} \\
127.4\end{array}$ & $\begin{array}{c}26.0 \mathrm{a} \\
21.1 \mathrm{a} \\
31.5\end{array}$ & $\begin{array}{c}7.5 \mathrm{a} \\
6.5 \mathrm{~b} \\
79.7 \\
\end{array}$ & $\begin{array}{c}60.8 \mathrm{a} \\
64.2 \mathrm{~b} \\
27.6 \\
\end{array}$ \\
\hline $40-60$ & $\begin{array}{c}\text { Row } 1 \\
\text { Row } 2 \\
\text { CV\% }\end{array}$ & $\begin{array}{c}8.4 \mathrm{a} \\
10.0 \mathrm{a} \\
25.9\end{array}$ & $\begin{array}{c}4.0 \mathrm{a} \\
4.0 \mathrm{a} \\
1.3\end{array}$ & $\begin{array}{c}0.9 \mathrm{a} \\
0.6 \mathrm{a} \\
64.8\end{array}$ & $\begin{array}{c}4.5 \mathrm{a} \\
4.8 \mathrm{a} \\
30.6\end{array}$ & $\begin{array}{c}0.6 \mathrm{a} \\
0.6 \mathrm{a} \\
52.0\end{array}$ & $\begin{array}{c}96.0 \mathrm{a} \\
101.4 \mathrm{a} \\
10.6\end{array}$ & $\begin{array}{c}16.9 \mathrm{a} \\
18.0 \mathrm{a} \\
12.2\end{array}$ & $\begin{array}{l}5.9 \mathrm{a} \\
6.0 \mathrm{a} \\
26.9\end{array}$ & $\begin{array}{c}3.4 \mathrm{a} \\
3.5 \mathrm{a} \\
48.6\end{array}$ & $\begin{array}{c}5.1 \mathrm{a} \\
3.5 \mathrm{a} \\
63.3\end{array}$ & $\begin{array}{c}26.4 \mathrm{a} \\
26.1 \mathrm{a} \\
25.2\end{array}$ & $\begin{array}{c}6.2 \mathrm{a} \\
6.1 \mathrm{a} \\
30.6\end{array}$ & $\begin{array}{c}65.1 \mathrm{a} \\
66.9 \mathrm{a} \\
10.9\end{array}$ \\
\hline
\end{tabular}

Transformations made: $\operatorname{DIFF}\left(\mathrm{pH} \_\mathrm{C}\right)$; $\mathrm{DIFF}\left(\mathrm{pH} \_\mathrm{D}\right)$; FIRST(pH_E); COSR(Ca_D); COSR(Ca_E); COSR(Ca_F);COSR(Mg_B);COSR(Mg_C); COSR(Mg_E); COSR(CEC_E); COSR(ECEC_D); COSR(ECEC_E); COSR(SB_D); COSR(SB_E); COSR(KECEC_D); COSR(CaECEC_A); COSR(CaECEC_D); COSR(CaECEC_E); COSR(MgECEC_A); COSR(MgECEC_B); COSR(V\%_D); FIRST(V\%_E); COSR(m\%_E). The presented means refer to the original values for each transformed variable, and the comparison of means test to the transformed variables. Means followed by the same letter for each layer do not differ from each other by the Tukey test when $\mathrm{p}<0.05$.

ECEC in the 20-40 cm layer (Table 3). Dose effects for $\mathrm{OC}$ in the layer of $10-15 \mathrm{~cm}$ and CaCEC in the layer of $5-10 \mathrm{~cm}$ were also observed (Table 4$)$.

\section{DISCUSSION}

\subsection{Soil attributes at 6 months}

Higher concentration of exchangeable $\mathrm{Mg}$ in the layer of 5-10 cm favored the higher SB in the same layer in row 2 . Higher $\mathrm{SB}$ in row 2 in the $0-5 \mathrm{~cm}$ and $5-10 \mathrm{~cm}$ layers at 6 months favored higher $\mathrm{V} \%$ in the same layers. On the other hand, the percentage of negative charged soil neutralized by basic reaction cations (V\%) in the $5-10 \mathrm{~cm}$ layer was below $45 \%$ (CQFSRS/SC, 2004).

Zaia and Gama-Rodriges (2004) found lower concentrations of $\mathrm{Ca}, \mathrm{Mg}$ and $\mathrm{K}$ in the soil under eucalyptus in relation to the present study; however, they were more acidic soils. An increase of $\mathrm{Mg}$ in row 2 at 6 months in the $5-10 \mathrm{~cm}$ and $15-20 \mathrm{~cm}$ layers was observed (Table 1), which corroborates with the highest concentration of $\mathrm{OC}$, although this was not significant since soil organic matter exchange (MOS) sites control bivalent cations such as $\mathrm{Ca}$ and $\mathrm{Mg}$ (Vitti et al., 2006). A higher concentration of $\mathrm{Mg}$ was only observed in row 1 for the $10-15 \mathrm{~cm}$ layer.

Linear adjustment with increasing doses of $\mathrm{K}$ was only observed for $\mathrm{K}$ in the $5-10 \mathrm{~cm}$ layer (Figure $1 \mathrm{~A}$ ), and for KECEC in the 0-5 cm layer (Figure 1B). As a result of the increasing responses of the $\mathrm{K}$ application, upon entering the system the fertilizers altered the composition of the solution, and consequently promoted changes in the chemical balance between the solid and liquid phases (Ernani et al., 2007). Average K concentrations (CQFSRS/SC, 2004) were verified up to $20-40 \mathrm{~cm}$ at 6 months (Table 1 ), and especially at 12 months (Table 2); however, this effect could not be observed at 30 months (Table 3 ).

There was a quadratic adjustment (Table 4) for CEC in the $20-40 \mathrm{~cm}$ layer with $\mathrm{K}$ doses, along with a higher CEC value at the dose of $200 \mathrm{~kg} \mathrm{ha}^{-1}$ year $^{-1}$. 
Table 3 - Average concentrations of soil chemical attributes in rows submitted to the $\mathrm{K}$ dose treatments 30 months after the start of the experiment.

Tabela 3 - Concentrações médias dos atributos químicos do solo nos renques submetidos aos tratamentos de doses de $K$, aos 30 meses após o início do experimento.

\begin{tabular}{|c|c|c|c|c|c|c|c|c|c|c|c|c|c|c|}
\hline Layer & Variation & $\mathrm{OC}$ & $\begin{array}{c}\mathrm{pH} \\
\mathrm{CaCl}_{2}\end{array}$ & $\mathrm{Ca}$ & $\mathrm{Mg}$ & $\mathrm{K}$ & CEC & ECEC & SB & KECEC & $\mathrm{CaECEC}$ & MgECEC & $\mathrm{V}$ & $\mathrm{m}$ \\
\hline $\mathrm{cm}$ & & $\mathrm{g} \mathrm{dm}^{-3}$ & & & \multicolumn{5}{|c|}{$\mathrm{mmol}_{\mathrm{c}} \mathrm{dm}^{-3}$} & \multicolumn{5}{|c|}{$\%$} \\
\hline \multirow[t]{3}{*}{$0-5$} & Row 1 & $23.4 \mathrm{a}$ & $4.7 \mathrm{a}$ & $46.7 \mathrm{a}$ & $29.9 \mathrm{a}$ & $1.3 \mathrm{a}$ & $150.9 \mathrm{a}$ & $79.4 \mathrm{a}$ & $78.0 \mathrm{a}$ & $1.8 \mathrm{a}$ & $60.8 \mathrm{a}$ & $35.6 \mathrm{a}$ & $51.1 \mathrm{a}$ & $1.9 \mathrm{a}$ \\
\hline & Row 2 & $24.0 \mathrm{a}$ & $4.7 \mathrm{a}$ & $45.4 \mathrm{a}$ & $35.4 \mathrm{a}$ & $1.3 \mathrm{a}$ & $156.1 \mathrm{a}$ & $83.8 \mathrm{a}$ & $82.0 \mathrm{a}$ & $1.6 \mathrm{a}$ & $56.8 \mathrm{a}$ & $39.6 \mathrm{a}$ & $52.1 \mathrm{a}$ & $2.0 \mathrm{a}$ \\
\hline & $\mathrm{CV} \%$ & 8.4 & 5.4 & 16.0 & 57.9 & 25.8 & 11.3 & 22.5 & 22.4 & 36.8 & 23.2 & 36.5 & 13.0 & 80.3 \\
\hline \multirow[t]{3}{*}{$5-10$} & Row 1 & $24.1 \mathrm{a}$ & $4.3 \mathrm{a}$ & $31.5 \mathrm{a}$ & $29.5 \mathrm{a}$ & $0.9 \mathrm{a}$ & $144.3 \mathrm{a}$ & $67.7 \mathrm{a}$ & $61.8 \mathrm{a}$ & $1.3 \mathrm{a}$ & $46.8 \mathrm{a}$ & $42.9 \mathrm{a}$ & $42.6 \mathrm{a}$ & $9.0 \mathrm{a}$ \\
\hline & Row 2 & $24.2 \mathrm{a}$ & $4.5 \mathrm{a}$ & $32.2 \mathrm{a}$ & $36.5 \mathrm{a}$ & $1.0 \mathrm{a}$ & $152.1 \mathrm{a}$ & $75.6 \mathrm{a}$ & $69.7 \mathrm{a}$ & $1.4 \mathrm{a}$ & $42.8 \mathrm{a}$ & $47.7 \mathrm{a}$ & $45.7 \mathrm{a}$ & $8.1 \mathrm{a}$ \\
\hline & $\mathrm{CV} \%$ & 5.0 & 6.0 & 23.3 & 30.4 & 33.2 & 11.4 & 20.2 & 21.9 & 35.7 & 18.9 & 17.4 & 15.7 & 44.0 \\
\hline \multirow[t]{3}{*}{$10-15$} & Row 1 & $18.3 \mathrm{a}$ & $4.0 \mathrm{~b}$ & $25.9 \mathrm{a}$ & $41.1 \mathrm{a}$ & $0.7 \mathrm{a}$ & $163.9 \mathrm{a}$ & $79.2 \mathrm{a}$ & $67.6 \mathrm{a}$ & $0.9 \mathrm{a}$ & $31.8 \mathrm{a}$ & $52.5 \mathrm{a}$ & $41.0 \mathrm{a}$ & $14.8 \mathrm{a}$ \\
\hline & Row 2 & $18.3 \mathrm{a}$ & $4.3 \mathrm{a}$ & $32.8 \mathrm{a}$ & $41.4 \mathrm{a}$ & $0.7 \mathrm{a}$ & $166.2 \mathrm{a}$ & $87.1 \mathrm{a}$ & $74.9 \mathrm{a}$ & $0.9 \mathrm{a}$ & $36.6 \mathrm{a}$ & $48.0 \mathrm{a}$ & $44.8 \mathrm{a}$ & $14.5 \mathrm{a}$ \\
\hline & $\mathrm{CV} \%$ & 8.0 & 6.0 & 45.8 & 23.4 & 40.8 & 10.7 & 18.9 & 21.6 & 49.6 & 31.7 & 21.2 & 13.8 & 27.2 \\
\hline \multirow[t]{3}{*}{$15-20$} & Row 1 & $17.0 \mathrm{a}$ & $4.0 \mathrm{a}$ & $12.5 \mathrm{a}$ & $44.6 \mathrm{a}$ & $0.7 \mathrm{a}$ & $159.1 \mathrm{a}$ & $70.6 \mathrm{a}$ & $57.7 \mathrm{a}$ & $1.0 \mathrm{a}$ & $17.8 \mathrm{a}$ & $62.8 \mathrm{a}$ & $36.1 \mathrm{a}$ & $18.4 \mathrm{a}$ \\
\hline & Row 2 & $18.3 \mathrm{a}$ & $4.1 \mathrm{a}$ & $11.6 \mathrm{a}$ & $53.4 \mathrm{a}$ & $0.8 \mathrm{a}$ & $167.8 \mathrm{a}$ & $78.1 \mathrm{a}$ & $65.7 \mathrm{a}$ & $1.0 \mathrm{a}$ & $15.0 \mathrm{a}$ & $67.9 \mathrm{a}$ & $39.1 \mathrm{a}$ & $16.1 \mathrm{a}$ \\
\hline & $\mathrm{CV} \%$ & 7.2 & 4.0 & 35.6 & 22.9 & 42.7 & 9.6 & 16.0 & 18.4 & 45.1 & 39.3 & 11.6 & 12.1 & 22.8 \\
\hline \multirow[t]{3}{*}{$20-40$} & Row 1 & $17.3 \mathrm{a}$ & $3.9 \mathrm{~b}$ & $4.0 \mathrm{a}$ & $21.1 \mathrm{a}$ & $0.7 \mathrm{a}$ & $122.9 \mathrm{~b}$ & $40.3 \mathrm{~b}$ & $25.7 \mathrm{a}$ & $1.7 \mathrm{a}$ & $10.1 \mathrm{a}$ & $51.6 \mathrm{a}$ & 20.9 a & $36.7 \mathrm{a}$ \\
\hline & Row 2 & $17.6 \mathrm{a}$ & $4.0 \mathrm{a}$ & $4.4 \mathrm{a}$ & $22.5 \mathrm{a}$ & $1.1 \mathrm{a}$ & $137.5 \mathrm{a}$ & $42.1 \mathrm{a}$ & $28.0 \mathrm{a}$ & $2.7 \mathrm{a}$ & $10.4 \mathrm{a}$ & $53.0 \mathrm{a}$ & $20.3 \mathrm{a}$ & $34.0 \mathrm{a}$ \\
\hline & $\mathrm{CV} \%$ & 9.0 & 5.3 & 22.9 & 21.4 & 57.5 & 8.1 & 13.2 & 19.2 & 57.5 & 21.6 & 10.4 & 17.1 & 13.4 \\
\hline \multirow[t]{3}{*}{$40-60$} & Row 1 & $17.4 \mathrm{a}$ & $4.1 \mathrm{a}$ & $3.3 \mathrm{a}$ & $13.9 \mathrm{a}$ & $0.9 \mathrm{a}$ & $117.1 \mathrm{a}$ & $30.0 \mathrm{a}$ & $18.2 \mathrm{a}$ & $3.0 \mathrm{a}$ & $11.0 \mathrm{a}$ & $46.2 \mathrm{a}$ & $15.5 \mathrm{a}$ & $39.8 \mathrm{a}$ \\
\hline & Row 2 & $17.5 \mathrm{a}$ & $4.1 \mathrm{a}$ & $4.2 \mathrm{a}$ & $15.6 \mathrm{a}$ & $0.8 \mathrm{a}$ & $125.1 \mathrm{a}$ & $32.2 \mathrm{a}$ & $20.5 \mathrm{a}$ & $2.6 \mathrm{a}$ & $12.7 \mathrm{a}$ & $48.0 \mathrm{a}$ & $16.4 \mathrm{a}$ & $36.8 \mathrm{a}$ \\
\hline & $\mathrm{CV} \%$ & 9.0 & 1.0 & 41.1 & 22.9 & 49.3 & 7.5 & 14.4 & 22.3 & 48.0 & 32.4 & 11.7 & 19.0 & 14.5 \\
\hline
\end{tabular}

Transformations made: CORS(pH_E); 1/(K_C); DIFF(K_D); DIFF(Ca_C); DIFF(Mg_D); CORS(CEC_B); CORS(CEC_D); COSR(SB_D); DIFF(KECEC_D); COSR(CaECEC_D); CORS(V\%_C). The presented means refer to the original values for each transformed variable, and the comparison of means test to the transformed variables. Means followed by the same letter for each layer do not differ from each other by the Tukey test when $\mathrm{p}<0.05$.

In addition, a negative quadratic fit (Table 4) was observed for $\mathrm{Mg}$ in the $0-5 \mathrm{~cm}$ layer. Therefore, the small effect of $\mathrm{K}$ doses after 6 months from the start of the experiment points to a short interval for evaluating such responses. Despite the higher KECEC in row 1 in the 40-60 cm layer, it was not possible to verify a standard behavior of KECEC between the rows at 6 months (Table 1).

Higher concentrations of $\mathrm{OC}$ at all evaluation times was observed in relation to the initial OC. This possibly occurred due to conservation management (branches remaining on the soil) in the rows, and the high relation $\mathrm{C}: \mathrm{N}$ of the eucalyptus litter (Gama-Rodrigues et al., 2008), thus minimizing decomposition by microorganisms, and gradually increasing OC reserves for sandy soils (Leite et al., 2010), as is the case of the present study.

Acidity reduction, especially of the rhizosphere, results in complexation and/or precipitation of the Al, thereby reducing the solubility and absorption, and consequently relieving the phytotoxic effects of this ion (Souza et al., 2011). This effect was clearly observed in the 0-5 cm layer with $\mathrm{pH} 5.0$ and $\mathrm{m} \%$ less than 1 , although it was not significant.

\subsection{Soil attributes at 12 months}

Linear fit of the interaction for ECEC and SB indicate higher concentrations in row 1 (Table 4) at 12 months in the $0-5 \mathrm{~cm}$ layer. On the other hand, quadratic reductions were observed for CaECEC with $\mathrm{K}$ doses in row 1 (Table 4) in the 20-40 cm layer, probably due to the competitive inhibition between $\mathrm{K}$ at high concentrations and $\mathrm{Ca}$ (Malavolta et al., 1997).

Lower $\mathrm{m} \%$ in the $20-40 \mathrm{~cm}$ layer in row 1 may be attributed to the higher $\mathrm{V} \%$ in the same layer. It is important to note that the high verified $\mathrm{m} \%$, mainly in the subsurface layers (15-60 cm, Table 2), is attributed to the higher Al content, as the $\mathrm{OC}$ reduction in the profile reduces complexation, with the exchangeable form predominating. However, eucalyptus does not have Al toxicity problems and the high $\mathrm{m} \%$ is not restrictive to growth (Bellote and Ferreira, 1993). Quadratic fit for K with doses was observed in the $0-5 \mathrm{~cm}$ layer, which favored linear fit

Revista Árvore. 2018;42(1):e420117 
Table 4 - Regression equations for concentration of macronutrients in different layers of soil, as affected by potassium rates and the interaction of the main factor (rows) and secondary (rates).

Tabela 4-Equações de regressão referentes às concentrações de macronutrientes em diferentes camadas de solo, em função de doses de potássio, e da interação do fator principal (renques) e secundário (doses).

\begin{tabular}{|c|c|c|c|c|c|}
\hline Attributes & Unit & Layer & Equation & $\mathrm{R}^{2}$ & $\mathrm{P}$ \\
\hline \multicolumn{6}{|c|}{6 months } \\
\hline $\mathrm{K}$ & $\mathrm{mmol}_{\mathrm{c}} \mathrm{dm}^{-3}$ & $5-10 \mathrm{~cm}$ & $y=0.0029 x+1.5097$ & 0.94 & 0.0298 \\
\hline $\mathrm{Mg}$ & $\mathrm{mmol}_{\mathrm{c}} \mathrm{dm}^{-3}$ & $0-5 \mathrm{~cm}$ & $y=-0.0001 x^{2}+0.0006 x+28.512$ & 0.99 & 0.0134 \\
\hline CEC & $\mathrm{mmol}_{\mathrm{c}} \mathrm{dm}^{-3}$ & $20-40 \mathrm{~cm}$ & $y=-0.0005 x^{2}+0.1898 x+124.9$ & 0.99 & 0.0253 \\
\hline KECEC & $\%$ & $0-5 \mathrm{~cm}$ & $\mathrm{y}=0.0062 \mathrm{x}+2.8898$ & 0.97 & 0.0145 \\
\hline \multicolumn{6}{|c|}{12 months } \\
\hline $\mathrm{K}$ & $\mathrm{mmol}_{\mathrm{c}} \mathrm{dm}^{-3}$ & $10-15 \mathrm{~cm}$ & $y=0.0042 x+1.2178$ & 0.95 & 0.0229 \\
\hline ECEC & $\mathrm{mmol}_{\mathrm{c}} \mathrm{dm}^{-3}$ & $0-5 \mathrm{~cm}$ & $y=0.0328 x+71.628$ & 0.92 & 0.039 \\
\hline SB & $\mathrm{mmol}_{\mathrm{c}} \mathrm{dm}^{-3}$ & $0-5 \mathrm{~cm}$ & $\mathrm{y}=0.0324 \mathrm{x}+71.003$ & 0.92 & 0.0417 \\
\hline KECEC & $\%$ & $10-15 \mathrm{~cm}$ & $y=-0.00002 x^{2}+0.0179 x+4.114$ & 0.99 & 0.0097 \\
\hline \multicolumn{6}{|c|}{30 months } \\
\hline $\mathrm{OC}$ & $\mathrm{g} \mathrm{dm}-3$ & $10-15 \mathrm{~cm}$ & $y=0.0001 x^{2}-0.0373 x+19.717$ & 0.99 & 0.0346 \\
\hline CaECEC & $\%$ & $5-10 \mathrm{~cm}$ & $y=-0.0004 x^{2}+0.1085 x+42.721$ & 0.99 & 0.0176 \\
\hline Attributes & Row & Layer & $\begin{array}{l}\text { Equation } \\
\end{array}$ & $\mathrm{R}^{2}$ & $\mathrm{P}$ \\
\hline \multicolumn{6}{|c|}{12 months } \\
\hline SB & 1 & $0-5 \mathrm{~cm}$ & $y=0.0559 x+65.54$ & 0.93 & 0.034 \\
\hline SB & 2 & $0-5 \mathrm{~cm}$ & $\wedge \bar{y}=y=77.8$ & - & - \\
\hline ECEC & 1 & $0-5 \mathrm{~cm}$ & $y=0.0553 x+66.363$ & 0.95 & 0.0243 \\
\hline ECEC & 2 & $0-5 \mathrm{~cm}$ & $\wedge \bar{y}=y=78.4$ & - & - \\
\hline CaECEC & 1 & $20-40 \mathrm{~cm}$ & $\mathrm{y}=-0.00001 \mathrm{x}^{2}-0.0001 \mathrm{x}+0.4387$ & 0.99 & 0.0479 \\
\hline CaECEC & 2 & $20-40 \mathrm{~cm}$ & $\wedge \bar{y}=y=9.17$ & - & - \\
\hline
\end{tabular}

for ECEC and SB and quadratic fit for KECEC in the same layer.

A linear effect for $\mathrm{K}$ in the $5-10 \mathrm{~cm}$ layer similarly favored KECEC in the same layer, as well as the linear effect for $\mathrm{K}$ in the $10-15 \mathrm{~cm}$ layer (Table 4) quadratically favored KECEC (Table 4). These K adjustments with $\mathrm{K}$ doses even occurred under good fertility conditions at 12 months (Table 2) (CQFSRS/SC, 2004). It is remarkable that $\mathrm{K}$ is mobile in the soil (Marschner, 2012) and decreasing $\mathrm{K}$ concentration in depth can be attributed to the use and rapid cycling by the root system. Due to eucalyptus roots distribution, which explores a considerable volume of soil through fine roots, it may have prevented nutrient losses through deep percolation (Laclau et al., 2013). No K leaching was observed in this study, even with $58 \%$ of sand in the $0-20 \mathrm{~cm}$ layer, as the applications were split in order to avoid large losses, as suggested by Costa et al. (2012).

\subsection{Soil attributes at 30 months}

The less acidic $\mathrm{pH}$ in row 2 favored higher CEC and ECEC in the $20-40 \mathrm{~cm}$ layer (Table 3 ). The same trend can be observed for $\mathrm{pH}$ in row 2 in the 10-15 $\mathrm{cm}$ layer. Quadratic fit of K doses were observed for OC in the 10-15 cm layer (Table 4), where the highest responses were found at the highest dose $\left(300 \mathrm{~kg} \mathrm{ha}^{-1}\right.$ year). Long-term fertilization influenced the increase of OC stock (Table 3). Such response may have contributed to CEC generation in soils under subtropical conditions (Raij, 1981), which affects cation retention and reduces leach losses. A CEC and ECEC increase was observed (Table 3 ) during the experiment in relation to 6 months (Table 1) because of conservation management of rows, thus corroborating with Grayston et al. (1996) who verified an increase in OC over time associated with eucalyptus presence.

Quadratic fits were also observed for CaECEC in the $5-10 \mathrm{~cm}$ layer (Table 4). However, lower doses of $\mathrm{K}$ provided the highest $\mathrm{CaECEC}$, although synergisms occur between $\mathrm{K}$ and $\mathrm{Ca}$ in low concentration (Malavolta et al., 1997).

Despite high KECEC at 6 and 12 months at close to $5 \%$ (Vitti et al., 2006), this situation was not repeated at 30 months, which represented less than $2 \%$ of the ECEC (Table 3). This reduction of KECEC was not 
expected at 30 months, since fertilization as well as the natural pruning were continuous. On the other hand, CaECEC up to $15 \mathrm{~cm}$ is adequate, which is between $35-45 \%$, but MgECEC is much larger than the appropriate range, between 5 and $20 \%$ (Vitti et al., 2006). This corroborates the low $\mathrm{Ca}: \mathrm{Mg}$ ratio in the profile at 30 months. Dijkstra and Fitzhugh (2003) found that Ca was the most important cation of CEC, and $\mathrm{K}$ represented less than $5 \%$ of ECEC, corroborating the results obtained here at 30 months (Table 3 ). On the other hand, the MgECEC was representatively higher than $\mathrm{Ca}$, which is not common since subtropical acid soils usually contain less $\mathrm{Mg}$ than $\mathrm{Ca}$, because $\mathrm{Mg}$ is not so strongly adsorbed by the clay and the SOM is more susceptible to leaching (Vitti et al., 2006; Salvador et al., 2011). Moreover, Marschner (2012) reported $5-10 \%$ of $\mathrm{Mg}$ from leaves is connected to pectates in the cell wall, which may be encouraging as the mineralization occurs, the $\mathrm{Mg}$ availability increases in the exchange complex.

In addition, the $\mathrm{K}$ recommendation for eucalyptus when the clay content is between $15-35 \%$ and $\mathrm{K}$ exchangeable between 1.5 and $3 \mathrm{mmol}_{\mathrm{c}} \mathrm{dm}^{-3}$ in the 0 $20 \mathrm{~cm}$ layer is $40 \mathrm{~kg}$ of $\mathrm{K}_{2} \mathrm{O}^{-1}$ (Silveira et al., 2005). In addition, $\mathrm{K}$ fertilization management in the present experiment focused on grain and forage production in ICLS, and even despite this, little fertilization response was observed since E. dunnii has a medium response to fertilization (Gonçalves and Mello, 2000). The results of soil with values above $1.0 \mathrm{mmol}_{\mathrm{c}} \mathrm{dm}^{-3}$ have been contradictory, since most of the time there are no responses to the application of this nutrient, and when they occur they are justified by the close $\mathrm{Ca}$ : $\mathrm{Mg}$ ratio ( $<1$ unit) or by the high values of $\mathrm{Ca}+\mathrm{Mg}$ in soil $\left(>8 \mathrm{mmolc} \mathrm{dm}^{-3}\right)$ (Silveira and Malavolta, 2000).

\section{CONCLUSIONS}

Conservationist management of rows favors the highest concentrations of exchangeable bases, particularly at 30 months of experimentation, contributing to increases in OC, CEC and ECEC, as well as increases in CaECEC, MgECEC and KECEC. Reduced exchangeable $\mathrm{K}$ at 30 months may be associated with the higher concentration of Mg in ECEC. High K mobility in depth was not verified, rejecting the hypothesis that there would be leaching. Little response to potassium fertilization was observed at macronutrient concentrations.

\section{REFERENCES}

Bellote AFJ, Ferreira CA. Nutrientes minerais e crescimento de árvores adubadas de Eucalyptus grandis, na região do cerrado, no estado de São Paulo. Boletim de Pesquisa Florestal. 1993(26/ 27):17-28.

Bernardino FS, Garcia R. Sistemas silvipastoris. Pesquisa Florestal Brasileira. 2009(60):77-87.

Cantarella H, Quaggio JÁ, Raij B van. Determinação da matéria orgânica. In: Raij B van, Andrade JC, Cantarella H, Quaggio JA, editores. Análise química para a avaliação da fertilidade de solos tropicais. Campinas: Instituto Agronômico de Campinas; 2001. p.173-80.

Comissão de Química e Fertilidade do Solo CQFSRS/SC. Manual de adubação e calagem para os Estados do Rio Grande do Sul e de Santa Catarina. $10^{\mathrm{a}}$.ed. Porto Alegre: Sociedade Brasileira de Ciência do Solo - Núcleo Regional Sul/UFRGS; 2004. 400p.

Costa MCG, Tonini H, Dias CTS, Iwata BF. Fertilization during the establishment of a Eucalyptus camaldulensis plantation in the northern Brazilian Amazon [internet]. Revista Agroambiente. 2012 6, n. 2, p. 91-101.

Cunha GM, Gama-Rodrigues AC, Costa GS. Ciclagem de nutrientes em Eucalyptus grandis W. Hill ex Maiden no norte fluminense. Revista Árvore. 2005;29(3):353-63.

Dijkstra FA, Fitzhugh RD. Aluminum solubility and mobility in relation to organic carbon in surface soils affected by six tree species of the northeastern United States. Geoderma. 2003;114(1/2):33-47.

Ernani PR, Almeida JA, Santos FC. Potássio. In: Novais RF, Alvarez V VH, Barros NF, Fontes RLF, Cantarutti RB, Neves JCL editores. Fertilidade do solo. Viçosa, MG: Sociedade Brasileira de Ciência do Solo; 2007. p.551-94.

Foster NW, Bhatti JS. Forest ecosystems: Nutrient cycling. In: Lal R editor. Encyclopedia of soil science. New York: Taylor; Francis Group; 2006. p.718-21.

Freycon V, Wonkam C, Fayolle A, Laclau JP, Lucot $\mathrm{E}$, Jourdan $\mathrm{C}$ et al. Tree roots can penetrate

Revista Árvore. 2018;42(1):e420117 
deeply in African semi-deciduous rain forests: evidence from two common soil types. Journal of Tropical Ecology. 2015;31(1):13-23.

Gama-Rodrigues EF, Barros NF, Viana AP, Santos GA. Alterações na biomassa e na atividade microbiana da serapilheira e do solo, em decorrência da substituição de cobertura florestal nativa por plantações de eucalipto, em diferentes sítios da região sudeste do Brasil. Revista Brasileira de Ciência do Solo. 2008;32(4):1489-99.

Grayston SJ, Vaughan D, Jones D. Rhizosphere carbon flow in trees, in comparison with annual plants: the importance of root exudation and its impact on microbial activity and nutrient availability. Applied Soil Ecology. 1996;5(1):29-56.

Gonçalves JLM, Mello SLM. O sistema radicular das árvores. In: Gonçalves JLM, Benedetti V editores. Nutrição e fertilização florestal. Piracicaba: IPEF; 2000. p.221-67.

Laclau J-P, Silva EA, Lambais GR, Bernoux M, Maire GL, Stape JL et al. Dynamics of soil exploration by fine roots down to a depth of 10 $\mathrm{m}$ throughout the entire rotation in Eucalyptus grandis plantations. Frontiers in Plant Science. 2013;4:1-12.

Leite FP, Silva IV, Novais RF, Barros NF. Neves JCL. Alterations of soil chemical properties by eucalyptus cultivation in five Regions in the Rio doce Valley. Revista Brasileira de Ciência do Solo. 2010;34(3):821-31.

Malavolta E. Potássio - Absorção, transporte e redistribuição na planta. In: Yamada T, Roberts TL. O potássio na agricultura brasileira. $3^{\mathrm{a}} \mathrm{ed}$. Piracicaba: Potafos; 2005. p.179-238.

Malavolta E, Vitti GC, Oliveira SA. Avaliação do estado nutricional das plantas: princípios e aplicações. $2^{\mathrm{a}}$ ed. Piracicaba: Potafos; 1997. 319p.

Marschner P. Mineral nutrition of higher plants. 3rd ed. London: Elsevier; 2012. 643p.

Menezes RSC, Salcedo I H, Elliott ET. Microclimate and nutrient dynamics in a silvopastoral system of semiarid northeastern Brazil. Agroforestry Systems. 2002;56(1):27-38
Oliveira RH, Rosolem CA, Trigueiro RM. Importância do fluxo de massa e difusão no suprimento de potássio ao algodoeiro como variável de água e potássio no solo. Revista Brasileira de Ciência do Solo. 2004;28(3):439-45.

Pavan MA, Bloch MF, Zempulski HC, Miyazawa M, Zocoler DC. Manual de análise química do solo e controle de qualidade. Londrina: IAPAR; 1992. 40p.

Raij B van, Feitosa CT, Cantarella H. A análise de solo para descriminar respostas à adubação para a cultura do milho. Bragantia. 1981;40(6):57-75.

Reissmann CB, Wisniewski C. Aspectos nutricionais de plantios de Pinus. In: Gonçalves JLM, Benedetti V editores. Nutrição e fertilização florestal. Piracicaba: IPEF; 2000. p.135-65.

Retzlaf JG, Stipp NAF, Archela E. Breve síntese geológica e geomorfológica da área do parque estadual do Guartelá no estado do Paraná. Geografia. 2006;15(1):95-106.

Salvador JT, Carvalho TC, Lucchesi LAC. Relações cálcio e magnésio presentes no solo e teores foliares de macronutrientes. Revista Acadêmica: Ciências Agrárias e Ambientais. 2011;9:27-32.

Silveira RLVA, Gava JL, Malavolta E. O potássio na cultura do eucalipto. In: Yamada T, Roberts TL editores. Potássio na agricultura brasileira. Piracicaba: Potafos; 2005. p.521-90.

Silveira RLV, Malavolta E. Nutrição e adubação potássica em Eucalyptus. Informações Agronômicas. 2000;91:1-12. (Encarte TécnicoPotafós).

Souza EP, Silva IF, Ferreira LE. Mecanismos de tolerância a estresses por metais pesados em plantas. Revista Brasileira de Agrociência. 2011;17(2-4):167-73.

Vitti GC, Lima E, Cicarone F. Cálcio, magnésio e enxofre. In: Fernandes MS editor. Nutrição mineral de plantas. Viçosa MG: Sociedade Brasileira de Ciência do Solo; 2006. p.300-25.

Witschoreck R, Schumacher MV, Caldeira MVW. 
Estimativa da biomassa e do comprimento de raízes finas em Eucalyptus urophylla S.T. Blake no município de Santa Maria-RS. Revista Árvore. 2003;27(2):177-83.
Zaia FC, Gama-Rodrigues AC. Ciclagem e balanço de nutrientes em povoamentos de eucalipto na região norte fluminense. Revista Brasileira de Ciência do Solo. 2004;28(5):843-52. 